\title{
AUTOMORPHISMS OF THE GENERALIZED QUOT SCHEMES
}

\author{
INDRANIL BISWAS AND SUKHENDU MEHROTRA
}

\begin{abstract}
Given a compact connected Riemann surface $X$ of genus $g \geq 2$, and integers $r \geq 2, d_{p}>0$ and $d_{z}>0$, in [BDHW], a generalized quot scheme $\mathcal{Q}_{X}\left(r, d_{p}, d_{z}\right)$ was introduced. Our aim here is to compute the holomorphic automorphism group of $\mathcal{Q}_{X}\left(r, d_{p}, d_{z}\right)$. It is shown that the connected component of $\operatorname{Aut}\left(\mathcal{Q}_{X}\left(r, d_{p}, d_{z}\right)\right)$ containing the identity automorphism is $\operatorname{PGL}(r, \mathbb{C})$. As an application of it, we prove that if the generalized quot schemes of two Riemann surfaces are holomorphically isomorphic, then the two Riemann surfaces themselves are isomorphic.
\end{abstract}

\section{INTRODUCTION}

In [BDHW], a generalized quot scheme was defined; we quickly recall it. Let $X$ be a compact connected Riemann surface of genus $g \geq 2$, and $r \geq 2, d_{p}>0$ and $d_{z}>0$ are integers. Let $\mathcal{Q}\left(\mathcal{O}_{X}^{\oplus r}, d_{p}\right)$ be the quot scheme that parametrizes the coherent subsheaves of $\mathcal{O}_{X}^{\oplus r}$ of rank $r$ and degree $-d_{p}$. This complex projective variety $\mathcal{Q}\left(\mathcal{O}_{X}^{\oplus r}, d_{p}\right)$ is a moduli space of vortices [BDW], [Br], [BR], [Ba], [EINOS]. This moduli space is extensively studied (cf. [Bif], [BGL]). The universal vector bundle over $X \times \mathcal{Q}\left(\mathcal{O}_{X}^{\oplus r}, d_{p}\right)$ will be denoted by $\mathcal{S}$. The generalized quot scheme $\mathcal{Q}_{X}\left(r, d_{p}, d_{z}\right)$ parametrizes torsionfree coherent sheaves $F$ on $X$ of rank $r$ and degree $d_{z}-d_{p}$ such that some member of the family $\mathcal{S}$ is a subsheaf of $F$. In [BDHW], the fundamental group and the cohomology of $\mathcal{Q}_{X}\left(r, d_{p}, d_{z}\right)$ were computed.

The natural action of $\mathrm{GL}(r, \mathbb{C})$ on the trivial vector bundle $\mathcal{O}_{X}^{\oplus r}$ produces a holomorphic action of $\operatorname{PGL}(r, \mathbb{C})$ on $\mathcal{Q}_{X}\left(r, d_{p}, d_{z}\right)$. The main result proved here says that $\operatorname{PGL}(r, \mathbb{C})$ is the connected component, containing the identity element, of the group of holomorphic automorphisms of $\mathcal{Q}_{X}\left(r, d_{p}, d_{z}\right)$; see Theorem 2.1.

Let $X^{\prime}$ be a compact connected Riemann surface of genus at least two. Fix positive integers $r^{\prime} \geq 2, d_{p}^{\prime}$ and $d_{z}^{\prime}$. Let $\mathcal{Q}_{X}^{\prime}\left(r^{\prime}, d_{p}^{\prime} . d_{z}^{\prime}\right)$ be the corresponding generalized quot scheme. As an application of Theorem 2.1, we prove the following (see Proposition 3.2):

Proposition 1.1. If the two varieties $\mathcal{Q}_{X}^{\prime}\left(r^{\prime}, d_{p}^{\prime} . d_{z}^{\prime}\right)$ and $\mathcal{Q}_{X}\left(r, d_{p}, d_{z}\right)$ are isomorphic, then $X$ is isomorphic to $X^{\prime}$.

\section{HolomorPhiC VECTOR FIELDS ON $\mathcal{Q}_{X}\left(r, d_{p}, d_{z}\right)$}

Let $X$ be a compact connected Riemann surface of genus $g$, with $g \geq 2$. Fix positive integers $r \geq 2, d_{p}$ and $d_{z}$. Let $\mathcal{Q}\left(\mathcal{O}_{X}^{\oplus r}, d_{p}\right)$ denote the quot scheme that parametrizes all the torsion quotients of $\mathcal{O}_{X}^{\oplus r}$ of degree $d_{p}$. Therefore, elements of $\mathcal{Q}\left(\mathcal{O}_{X}^{\oplus r}, d_{p}\right)$ represent

2010 Mathematics Subject Classification. 14H60, 14D21, 14D23.

Key words and phrases. Generalized quot scheme, vector fields, automorphism group, symmetric product. 
subsheaves $S$ of $\mathcal{O}_{X}^{\oplus r}$ such that $\operatorname{rank}(S)=r$ and $\operatorname{degree}(S)=-d_{p}$. These two conditions on the subsheaf $S$ are together equivalent to the condition that $\mathcal{O}_{X}^{\oplus r} / S$ is torsion of degree $d_{p}$. There is a universal short exact sequence of sheaves on $X \times \mathcal{Q}\left(\mathcal{O}_{X}^{\oplus r}, d_{p}\right)$

$$
0 \longrightarrow \mathcal{S} \longrightarrow p_{X}^{*} \mathcal{O}_{X}^{\oplus r}=\mathcal{O}_{X \times \mathcal{Q}\left(\mathcal{O}_{X}^{\oplus r}, d_{p}\right)}^{\oplus r} \longrightarrow \mathcal{T}_{1} \longrightarrow 0
$$

where $p_{X}: X \times \mathcal{Q}\left(\mathcal{O}_{X}^{\oplus r}, d_{p}\right) \longrightarrow X$ is the natural projection.

Let

$$
f: \mathcal{Q}=\mathcal{Q}_{X}\left(r, d_{p}, d_{z}\right) \longrightarrow \mathcal{Q}\left(\mathcal{O}_{X}^{\oplus r}, d_{p}\right)
$$

be the relative quot scheme that parametrizes torsion quotients of $\mathcal{S}^{*}$ of degree $d_{z}$ (see (2.1) ). In other words, if $z$ is the point of $\mathcal{Q}\left(\mathcal{O}_{X}^{\oplus r}, d_{p}\right)$ representing a subsheaf $S \subset \mathcal{O}_{X}^{\oplus r}$, then the fiber $f^{-1}(z)$ is the the space of all subsheaves of $S^{*}$ of rank $r$ and degree $d_{p}-d_{z}$. Note that the degree of $S^{*}$ is $d_{p}$. Therefore, elements of $\mathcal{Q}$ parametrize diagrams of the form

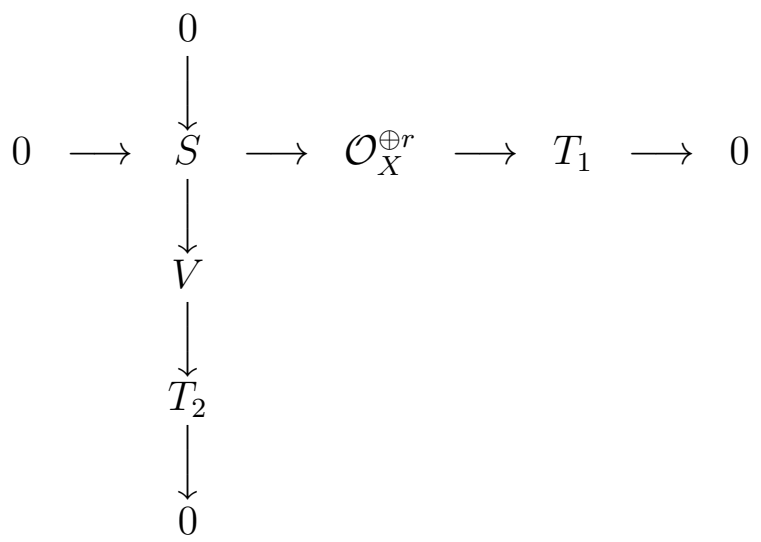

where $T_{1}$ and $T_{2}$ are torsion sheaves of degree $d_{p}$ and $d_{z}$ respectively, and the subsheaf $S \subset \mathcal{O}_{X}^{\oplus r}$ corresponds to the image, under $f$, of the point of $\mathcal{Q}$ corresponding to $V$.

Let $\operatorname{Aut}(\mathcal{Q})$ denote the group of all holomorphic automorphisms of $\mathcal{Q}$. Since $\mathcal{Q}$ is a smooth complex projective variety, its holomorphic automorphisms are automatically algebraic. Thus $\operatorname{Aut}(\mathcal{Q})$ is a complex Lie group with Lie algebra $H^{0}(\mathcal{Q}, T \mathcal{Q})$, where $T \mathcal{Q}$ is the holomorphic tangent bundle of $\mathcal{Q}$; the Lie algebra structure is given by the Lie bracket operation of vector fields. Let

$$
\operatorname{Aut}^{0}(\mathcal{Q}) \subset \operatorname{Aut}(\mathcal{Q})
$$

be the connected component containing the identity element.

The standard action of $\mathrm{GL}(r, \mathbb{C})$ on $\mathbb{C}^{r}$ produces an action of $\mathrm{GL}(r, \mathbb{C})$ on $\mathcal{O}_{X}^{\oplus r}$, because the total space of $\mathcal{O}_{X}^{\oplus r}$ is identified with $X \times \mathbb{C}^{r}$. This action of $\operatorname{GL}(r, \mathbb{C})$ on $\mathcal{O}_{X}^{\oplus r}$ defines an action of $\mathrm{GL}(r, \mathbb{C})$ on $\mathcal{Q}\left(\mathcal{O}_{X}^{\oplus r}, d_{p}\right)$. This action of $\mathrm{GL}(r, \mathbb{C})$ on $\mathcal{Q}\left(\mathcal{O}_{X}^{\oplus r}, d_{p}\right)$ evidently lifts to an action of $\mathrm{GL}(r, \mathbb{C})$ on $\mathcal{Q}$ (see (2.2)). Indeed, $\mathrm{GL}(r, \mathbb{C})$ acts on diagrams of type $(2.3)$. Since $\mathrm{GL}(r, \mathbb{C})$ is connected, we get a homomorphism

$$
\mathrm{GL}(r, \mathbb{C}) \longrightarrow \operatorname{Aut}^{0}(\mathcal{Q})
$$

The center $\mathbb{C}^{\star}=\mathbb{C} \backslash\{0\}$ of $\mathrm{GL}(r, \mathbb{C})$ acts trivially on $\mathcal{Q}$. Hence the above homomorphism produces a homomorphism

$$
\varphi: \operatorname{PGL}(r, \mathbb{C}) \longrightarrow \operatorname{Aut}^{0}(\mathcal{Q})
$$

Theorem 2.1. The homomorphism $\varphi$ in (2.4) is an isomorphism. 
Proof. Let

$$
p: \mathcal{Q} \longrightarrow \operatorname{Sym}^{d_{p}}(X) \times \operatorname{Sym}^{d_{z}}(X)
$$

be the morphism that sends any $z \in \mathcal{Q}$ to the support of $T_{1}$ with multiplicity given by $T_{1}$ and the support of $T_{2}$ with multiplicity given by $T_{2}$, where $T_{1}$ and $T_{2}$ are the torsion sheaves in the diagram (2.3) corresponding to the point $z$.

The homomorphism $\varphi$ is injective because the homomorphism

$$
\operatorname{PGL}(r, \mathbb{C}) \longrightarrow \operatorname{Aut}\left(\mathbb{C P}^{r-1}\right)
$$

given by the standard action of $\mathrm{GL}(r, \mathbb{C})$ on $\mathbb{C}^{r}$ is injective. Indeed, for any

$$
x=\left(\left(x_{1}, \cdots, x_{d_{p}}\right),\left(y_{1}, \cdots, y_{d_{z}}\right)\right) \in \operatorname{Sym}^{d_{p}}(X) \times \operatorname{Sym}^{d_{z}}(X),
$$

where all the above $d_{p}+d_{z}$ points are distinct, the fiber $p^{-1}(x)$ is $\left(\mathbb{C P} \mathbb{P}^{r-1}\right)^{d_{p}} \times\left(C P^{r-1}\right)^{d_{z}}$ (see $(2.5)$ ), and the action of $\operatorname{PGL}(r, \mathbb{C})$ on $p^{-1}(x)$ coincides with the diagonal action of $\operatorname{PGL}(r, \mathbb{C})$ on the factors in the above Cartesian product.

We need to prove that $\varphi$ is surjective.

The Lie algebra of $\operatorname{PGL}(r, \mathbb{C})$ will be denoted by $\mathfrak{g}$; it is the Lie algebra structure on trace zero $r \times r$-matrices with complex entries given by commutator. Let

$$
\theta: \mathfrak{g} \longrightarrow H^{0}(\mathcal{Q}, T \mathcal{Q})
$$

be the homomorphism of Lie algebras corresponding to the homomorphism $\varphi$ in (2.4). To prove that $\varphi$ is surjective, it suffices to show that $\theta$ is surjective.

The following lemma is a key step in the computation of $H^{0}(\mathcal{Q}, T \mathcal{Q})$.

Lemma 2.2. The Lie algebra $H^{0}(\mathcal{Q}, T \mathcal{Q})$ has a natural injective homomorphism to $\mathfrak{g} \oplus \mathfrak{g}$.

Proof of Lemma 2.2. For any positive integer $k$, let $P(k)$ denote the group of all permutations of $\{1, \cdots, k\}$. Consider the action of $P\left(d_{p}\right) \times P\left(d_{z}\right)$ on $X^{d_{p}+d_{z}}$ that permutes the first $d_{p}$ factors and the last $d_{z}$ factors. The quotient $X^{d_{p}+d_{z}} /\left(P\left(d_{p}\right) \times P\left(d_{z}\right)\right)$ is $\operatorname{Sym}^{d_{p}}(X) \times \operatorname{Sym}^{d_{z}}(X)$. Let

$$
q: X^{d_{p}+d_{z}} \longrightarrow \operatorname{Sym}^{d_{p}}(X) \times \operatorname{Sym}^{d_{z}}(X)
$$

be the corresponding quotient map.

Let

$$
\widetilde{U} \subset X^{d_{p}+d_{z}}
$$

be the complement of the big diagonal, so $\widetilde{U}$ parametrizes all possible distinct $d_{p}+d_{z}$ ordered points of $X$. The image $q(\widetilde{U}) \subset \operatorname{Sym}^{d_{p}}(X) \times \operatorname{Sym}^{d_{z}}(X)$ will be denoted by $U$. Since $p^{-1}(U)$ is a Zariski dense open subset of $\mathcal{Q}$, where $p$ is defined in (2.5), we have

$$
H^{0}(\mathcal{Q}, T \mathcal{Q}) \subset H^{0}\left(p^{-1}(U), T\left(p^{-1}(U)\right)\right) .
$$

The Galois group $\Gamma:=P\left(d_{p}\right) \times P\left(d_{z}\right)$ for the étale covering

$$
\left.q\right|_{\widetilde{U}}: \widetilde{U} \longrightarrow U
$$

where $\widetilde{U}$ is defined in (2.7), acts on the fiber product $\mathcal{Z}:=p^{-1}(U) \times_{U} \widetilde{U}$. We have

$$
H^{0}\left(p^{-1}(U), T \mathcal{Q}\right)=H^{0}(\mathcal{Z}, T \mathcal{Z})^{\Gamma},
$$


because the projection $\mathcal{Z} \longrightarrow p^{-1}(U)$ to the first factor of the fiber product is an étale Galois covering with Galois group $\Gamma$.

Now we have $\mathcal{Z}=\widetilde{U} \times\left(\mathbb{C} P^{r-1}\right)^{d_{p}} \times\left(\mathbb{C} P^{r-1}\right)^{d_{z}}$, where $\mathbb{C} P^{r-1}$ is the projective space parametrizing the lines in $\mathbb{C}^{r}$. Note that

$$
H^{0}\left(\mathbb{C P}^{r-1}, T \mathbb{C} P^{r-1}\right)=\mathfrak{g}=H^{0}\left(\mathbb{C} P^{r-1}, T \mathbb{C} P^{r-1}\right) .
$$

It is known that $H^{0}(\widetilde{U}, T \widetilde{U})=0[\mathrm{BDH}$, p. 1452, Proposition 2.3]. Also, we have $H^{0}\left(\widetilde{U}, \mathcal{O}_{\widetilde{U}}\right)=0[\underline{\mathrm{BDH}}$, p. 1449, Lemma 2.2]. These together imply that

$$
H^{0}(\mathcal{Z}, T \mathcal{Z})=\mathfrak{g}^{\oplus d_{p}} \oplus \mathfrak{g}^{\oplus d_{z}} .
$$

The action of $\Gamma=P\left(d_{p}\right) \times P\left(d_{z}\right)$ on $\mathfrak{g}^{\oplus d_{p}} \oplus \mathfrak{g}^{\oplus d_{z}}=H^{0}(\mathcal{Z}, T \mathcal{Z})$ (see (2.9) ) is the one that permutes first $d_{p}$ factors and the last $d_{z}$ factors. Hence we have $H^{0}(\mathcal{Z}, T \mathcal{Z})^{\Gamma}=\mathfrak{g} \oplus \mathfrak{g}$. Therefore,

$$
H^{0}\left(p^{-1}(U), T\left(p^{-1}(U)\right)\right)=\mathfrak{g} \oplus \mathfrak{g}
$$

by (2.9). Now the lemma follow from (2.8).

Next we will need a property of the Hecke transformations.

Let $Y$ be a smooth complex algebraic curve and $y_{0} \in Y$ a point; the curve $Y$ need not be projective. Fix a linear nonzero proper subspace $0 \neq S \subsetneq \mathbb{C}^{r}$. Consider the short exact sequence of sheaves on $Y$

$$
0 \longrightarrow V \longrightarrow \mathcal{O}_{Y}^{\oplus r} \longrightarrow \mathcal{O}_{y_{0}}^{\oplus r} / S=\mathbb{C}^{r} / S \longrightarrow 0 \text {. }
$$

Let $P(V)$ denote the projective bundle over $Y$ that parametrizes the lines in the fibers of $V$. Take any $A \in \mathrm{GL}(r, \mathbb{C})$. Let $\widehat{A}$ be the automorphism of $P\left(\mathcal{O}_{Y}^{\oplus r}\right)=Y \times C P^{r-1}$ given by $A$; this automorphism acts trivially on $Y$ and has the standard action on $C P^{r-1}$. Since $V$ and $\mathcal{O}_{Y}^{\oplus r}$ are identified over $Y \backslash\left\{y_{0}\right\}$, the above automorphism $\widehat{A}$ produces an automorphism of $\left.P(V)\right|_{Y \backslash\left\{y_{0}\right\}}$. This automorphism of $\left.P(V)\right|_{Y \backslash\left\{y_{0}\right\}}$ will be denoted by $\widehat{A}^{\prime}$.

Lemma 2.3. The above automorphism $\widehat{A}^{\prime}$ of $\left.P(V)\right|_{Y \backslash\left\{y_{0}\right\}}$ extends to a self-map of $P(V)$ if and only if $A(S)=S$.

Proof of Lemma 2.3. Let GL $(V)$ be the Zariski open subset of the total space of $\operatorname{End}(V)=$ $V \otimes V^{*}$ parametrizing endomorphisms of fibers that are automorphisms. The quotient $\operatorname{PGL}(V)=\mathrm{GL}(V) / \mathbb{C}^{*}$ is a group-scheme over $Y$ with fibers isomorphic to the group $\operatorname{PGL}(r, \mathbb{C})$. If an algebraic map of the total space

$$
\tau: P(V) \longrightarrow P(V)
$$

is an automorphism satisfies the condition that there is a nonempty Zariski open subset $U_{\tau} \subset Y$ such that $\tau$ restricts to an automorphism of $\left.P(V)\right|_{U_{\tau}}$ over the identity map of $U_{\tau}$, then $\tau$ is actually an automorphism over the identity map of $Y$. We note that the group of automorphisms of $P(V)$ over the identity map of $Y$ is precisely the group of sections, over $Y$, of the group-scheme $\operatorname{PGL}(V)$.

Fix a subspace $S^{\prime} \subset \mathbb{C}^{r}$ complementary to $S$, so $\mathbb{C}^{r}=S \oplus S^{\prime}$. Let $E_{S}:=Y \times S$ and $E_{S^{\prime}}:=Y \times S^{\prime}$ be the trivial algebraic vector bundles over $Y$ with fibers $S$ and $S^{\prime}$ respectively. Then we have

$$
\mathcal{O}_{Y}^{\oplus r}=E_{S} \oplus E_{S^{\prime}} \quad \text { and } V=E_{S} \oplus\left(E_{S^{\prime}} \otimes \mathcal{O}_{Y}\left(-y_{0}\right)\right) .
$$


From the above decompositions it follows immediately that if $A(S)=S$, then $\widehat{A}^{\prime}$ extends to an automorphism of $P(V)$.

To prove the converse, assume that $\widehat{A}^{\prime}$ extends to an automorphism of $P(V)$. It suffices to show that the subbundle $E_{S} \subset V$ in (2.12) is preserved by the section of $\operatorname{PGL}(V)$ corresponding to the automorphism of $P(V)$. Note that the restriction of this section of $\operatorname{PGL}(V)$ to $Y \backslash\left\{y_{0}\right\}$ is given by $A$. There is no nonzero homomorphism from $E_{S}$ to $E_{S^{\prime}} \otimes \mathcal{O}_{Y}\left(-y_{0}\right)$ which is given by a constant homomorphism $B: S \longrightarrow S^{\prime}$ on $Y \backslash\left\{y_{0}\right\}$ because such a homomorphism over $Y \backslash\left\{y_{0}\right\}$ extends to a homomorphism from $\left(E_{S}\right)_{y_{0}}$ to $\left(E_{S^{\prime}}\right)_{y_{0}}$ and this homomorphism $\left(E_{S}\right)_{y_{0}} \longrightarrow\left(E_{S^{\prime}}\right)_{y_{0}}$ coincides with $B$. On the other hand, the image of $\left(E_{S^{\prime}} \otimes \mathcal{O}_{Y}\left(-y_{0}\right)\right)_{y_{0}}$ in $\left(E_{S^{\prime}}\right)_{y_{0}}$ is the zero subspace. So if $B \neq 0$, then the homomorphism over $Y \backslash\left\{y_{0}\right\}$ does not extend to a homomorphism from $E_{S}$ to $E_{S^{\prime}} \otimes \mathcal{O}_{Y}\left(-y_{0}\right)$ over $Y$. Therefore, we conclude that $A(S)=S$.

Fix distinct $d_{p}-1$ points $x_{1}, \cdots, x_{d_{p}-1}$ on $X$. For each $x_{i}$, fix a hyperplane $H_{i}$ in $\left(\mathcal{O}_{X}^{\oplus r}\right)_{x_{i}}=\mathbb{C}^{r}$. Also, fix distinct $d_{z}-1$ points $y_{1}, \cdots, y_{d_{z}-1}$ on $X$ such that $x_{i} \neq y_{j}$ for all $1 \leq i \leq d_{p}-1$ and $1 \leq j \leq d_{z}-1$. Fix a line $L_{j}$ in $\left(\mathcal{O}_{X}^{\oplus r}\right)_{y_{j}}=\mathbb{C}^{r}$ for each $j$.

Now take the complement $Y=X \backslash\left\{x_{1}, \cdots, x_{d_{p}-1}, y_{1}, \cdots, y_{d_{z}-1}\right\}$. Take any nontrivial element

$$
\text { Id } \neq A \in \operatorname{PGL}(r, \mathbb{C}) .
$$

Fix a point $x_{0} \in Y$ and also fix a hyperplane

$$
S \subset\left(\mathcal{O}_{X}^{\oplus r}\right)_{x_{0}}=\mathbb{C}^{r}
$$

such that

$$
A(S) \neq S
$$

since $A \neq \mathrm{Id}$, such a subspace exists. Consider the vector bundle $V$ on $Y$ constructed in (2.11) using $S$. As before, $P(V)$ denotes the projective bundle over $Y$ parametrizing the lines in the fibers of $V$.

There is an embedding

$$
\delta: P(V) \longrightarrow \mathcal{Q}
$$

which we will now describe. For the map $f$ in (2.2), the image $f \circ \delta(P(V))$ is the point given by the quotient

$$
\left.0 \longrightarrow \widehat{V} \longrightarrow \mathcal{O}_{X}^{\oplus r} \longrightarrow\left(\oplus_{i=1}^{d_{p}-1}\left(\mathcal{O}_{X}^{\oplus r}\right)_{x_{i}} / H_{i}\right) \oplus\left(\mathcal{O}_{X}^{\oplus r}\right)_{x_{0}} / S\right) \longrightarrow 0,
$$

where $H_{i}$ are the hyperplanes fixed above; in particular, $f \circ \delta$ is a constant map. Note that $\widehat{V}$ is an extension of the vector bundle $V$ to $X$. For any point $y \in Y$ and any point in the fiber $y^{\prime} \in P(V)_{y}$, consider the short exact sequence on $X$

$$
0 \longrightarrow E \longrightarrow \widehat{V}^{*} \longrightarrow\left(\oplus_{j=1}^{d_{z}-1}\left(\widehat{V}_{y_{j}}^{*} / L_{j}^{\perp}\right) \oplus\left(\widehat{V}_{y}^{*} / L\left(y^{\prime}\right)^{\perp}\right) \longrightarrow 0\right.
$$

where $L\left(y^{\prime}\right) \subset V_{y}$ is the line in $V_{y}$ corresponding to the above point $y^{\prime}$, and $L_{j}^{\perp} \subset \widehat{V}_{y_{j}}^{*}$ (respectively, $L\left(y^{\prime}\right)^{\perp} \subset \widehat{V}_{y}^{*}$ ) is the annihilator of $L_{j}$ (respectively, $L\left(y^{\prime}\right)$ ); note that $\widehat{V}_{y_{j}}$ is identified with $\mathbb{C}^{r}$ and $\widehat{V}_{y}$ is identified with $V_{y}$. Therefore, we have

$$
\widehat{V} \hookrightarrow E^{*} \text {. }
$$

The map $\delta$ in (2.15) sends any $y^{\prime}$ to the above extension $E^{*}$ of $\widehat{V}$ constructed from $y^{\prime}$. 
From (2.10) we know that $\operatorname{PGL}(r, \mathbb{C})$ is contained in $\operatorname{Aut}\left(p^{-1}(U)\right)$ with

$$
0 \oplus \mathfrak{g} \subset \mathfrak{g} \oplus \mathfrak{g}=H^{0}\left(p^{-1}(U), T\left(p^{-1}(U)\right)\right)
$$

as its Lie algebra. This action of $\operatorname{PGL}(r, \mathbb{C})$ on $p^{-1}(U)$ clearly preserves the intersection $\delta(P(V)) \cap p^{-1}(U)$. Therefore, if the action of the element $A$ in (2.13) extends to $\mathcal{Q}$, then the extended action must preserve the image $\delta(P(V))$.

On the other hand, from Lemma 2.3 we know that the action of $A$ on $\left.P(V)\right|_{Y \backslash\left\{y_{0}\right\}}$ does not extend to $P(V)$ because (2.14) holds. This completes the proof of the theorem.

\section{HOLOMORPHIC MAPS FROM A SYMMETRIC PRODUCT}

Proposition 3.1. Let $X$ and $Y$ be compact connected Riemann surface with

$$
\operatorname{genus}(X) \geq \operatorname{genus}(Y) \geq 2 .
$$

If there is a nonconstant holomorphic map $\beta: \operatorname{Sym}^{d}(Y) \longrightarrow X$, then $d=1$, and $\beta$ is an isomorphism.

Proof. Let $\beta: \operatorname{Sym}^{d}(Y) \longrightarrow X$ be a nonconstant holomorphic map. Let

$$
\beta^{*}: H^{0}\left(X, \Omega_{X}^{1}\right) \longrightarrow H^{0}\left(\operatorname{Sym}^{d}(Y), \Omega_{\operatorname{Sym}^{d}(Y)}^{1}\right)
$$

be the pull-back of 1 -forms defined by $\omega \longmapsto \beta^{*} \omega$. This homomorphism $\beta^{*}$ is injective, because $\beta$ is surjective. Since

$$
\operatorname{dim} H^{0}\left(\operatorname{Sym}^{d}(Y), \Omega_{\operatorname{Sym}^{d}(Y)}^{1}\right)=\operatorname{genus}(Y)
$$

[Ma, p. 322, (4.3)], the injectivity of $\beta^{*}$ implies that $\operatorname{genus}(Y) \geq \operatorname{genus}(X)$. Therefore, the given condition genus $(X) \geq \operatorname{genus}(Y)$ implies that

- $\operatorname{genus}(X)=\operatorname{genus}(Y)$, and

- the above homomorphism $\beta^{*}$ is an isomorphism.

If $d \geq 2$, the wedge product

$$
\wedge^{2} H^{0}\left(\operatorname{Sym}^{d}(Y), \Omega_{\operatorname{Sym}^{d}(Y)}^{1}\right) \longrightarrow H^{0}\left(\operatorname{Sym}^{d}(Y), \Omega_{\operatorname{Sym}^{d}(Y)}^{2}\right)
$$

is a nonzero homomorphism [Ma, p. 325, (6.3)]. On the other hand, the wedge product on $H^{0}\left(X, \Omega_{X}^{1}\right)$ is the zero homomorphism because $H^{0}\left(X, \Omega_{X}^{2}\right)=0$. In other words, $\beta^{*}$ is not compatible with the wedge product operation on holomorphic 1 -forms if $d \geq 2$. So we conclude that $d=1$.

Since genus $(X)=\operatorname{genus}(Y)$, from Riemann-Hurwitz formula for Euler characteristic if follows that $\operatorname{degree}(\beta)=1$. In other words, $\beta$ is an isomorphism.

Let $X^{\prime}$ be a compact connected Riemann surface of genus at least two. Fix positive integers $r^{\prime} \geq 2, d_{p}^{\prime}$ and $d_{z}^{\prime}$. Let

$$
\mathcal{Q}^{\prime}=\mathcal{Q}_{X}^{\prime}\left(r^{\prime}, d_{p}^{\prime} \cdot d_{z}^{\prime}\right)
$$

be the corresponding generalized quot scheme (see (2.2) ).

Proposition 3.2. If the two varieties $\mathcal{Q}^{\prime}$ and $\mathcal{Q}$ (constructed in (2.2)) are isomorphic, then $X$ is isomorphic to $X^{\prime}$. 
Proof. Assume that $\mathcal{Q}^{\prime}$ and $\mathcal{Q}$ are isomorphic. We will show that $X$ and $X^{\prime}$ are isomorphic. Let $\eta: \operatorname{Sym}^{d_{p}}(X) \times \operatorname{Sym}^{d_{z}}(X) \longrightarrow \operatorname{Pic}^{d_{p}}(X) \times \operatorname{Pic}^{d_{z}}(X)$ be the morphism defined by

$$
\left(\left(x_{1}, \cdots, x_{d_{p}}\right),\left(y_{1}, \cdots, y_{d_{z}}\right)\right) \longmapsto\left(\mathcal{O}_{X}\left(x_{1}+\ldots+x_{d_{p}}\right), \mathcal{O}_{X}\left(y_{1}+\ldots+y_{d_{z}}\right)\right) .
$$

Since the general fiber of the map $p$ in (2.5) is a product of copies of projective spaces, the composition

$$
\eta \circ p: \mathcal{Q} \longrightarrow \operatorname{Pic}^{d_{p}}(X) \times \operatorname{Pic}^{d_{z}}(X)
$$

is the Albanese map for $\mathcal{Q}$, as there is no nonconstant holomorphic map from a projective space to an abelian variety. In particular, the Albanese variety of $\mathcal{Q}$ is of dimension $2 g=2 \cdot \operatorname{genus}(X)$. Therefore, comparing the Albanese varieties of $\mathcal{Q}$ and $\mathcal{Q}^{\prime}$ we conclude that $\operatorname{genus}(X)=g=\operatorname{genus}\left(X^{\prime}\right)$.

Fix a maximal torus $T$ in $\operatorname{Aut}^{0}(\mathcal{Q})$. In view of Theorem 2.1, this amounts to choosing a trivialization of $\mathcal{O}_{X}^{\oplus r}$, with two trivializations being identified if they differ by multiplication with a constant nonzero scalar. The fixed-point locus

$$
\mathcal{Q}^{T} \subset \mathcal{Q},
$$

for the action of $T$ on $\mathcal{Q}$, is a disjoint union of copies of

$$
\left(\operatorname{Sym}^{a_{1}}(X) \times \cdots \times \operatorname{Sym}^{a_{r}}(X)\right) \times\left(\operatorname{Sym}^{b_{1}}(X) \times \cdots \times \operatorname{Sym}^{b_{r}}(X)\right)
$$

with $\sum_{i=1}^{r} a_{i}=d_{p}$ and $\sum_{i=1}^{r} b_{i}=d_{z}$.

Take a component

$$
Z=\left(\operatorname{Sym}^{a_{1}}(X) \times \cdots \times \operatorname{Sym}^{a_{r}}(X)\right) \times\left(\operatorname{Sym}^{b_{1}}(X) \times \cdots \times \operatorname{Sym}^{b_{r}}(X)\right)
$$

of $\mathcal{Q}^{T}$ such that at least one of the $2 r$ integers $\left\{a_{1}, \cdots, a_{r}, b_{1}, \cdots, b_{r}\right\}$ is one.

We will first show that $Z$ is not holomorphically isomorphic to $\operatorname{Sym}^{c_{1}}(Y) \times \cdots \times$ $\operatorname{Sym}^{c_{n}}(Y)$, where $Y$ is compact connected Riemann surface of genus $g$ and $c_{j} \geq 2$ for all $1 \leq j \leq n$. To prove this, assume that $Z$ is isomorphic to $\operatorname{Sym}^{c_{1}}(Y) \times \cdots \times \operatorname{Sym}^{c_{n}}(Y)$, where $Y$ and $c_{i}$ are as above. Consider the composition

$$
\operatorname{Sym}^{c_{1}}(Y) \times \cdots \times \operatorname{Sym}^{c_{n}}(Y) \stackrel{\sim}{\longrightarrow} Z \stackrel{q}{\longrightarrow} X
$$

where $q$ is the projection to a factor of $Z$ which is the first symmetric power of $X$ (it is assumed that such a factor exists). Since all $c_{j} \geq 2$, from Proposition 3.1 it follows that there is no nonconstant map from $\operatorname{Sym}^{c_{1}}(Y) \times \cdots \times \operatorname{Sym}^{c_{n}}(Y)$ to $X$. Therefore, we conclude that $Z$ is not holomorphically isomorphic to $\operatorname{Sym}^{c_{1}}(Y) \times \cdots \times \operatorname{Sym}^{c_{n}}(Y)$.

Fix a maximal torus $T^{\prime} \subset \operatorname{Aut}\left(\mathcal{Q}^{\prime}\right)^{0}$. Since $\mathcal{Q}^{\prime}$ is isomorphic to $\mathcal{Q}$, there is a component

$$
\left(\operatorname{Sym}^{a_{1}^{\prime}}\left(X^{\prime}\right) \times \cdots \times \operatorname{Sym}^{a_{r}^{\prime}}\left(X^{\prime}\right)\right) \times\left(\operatorname{Sym}^{b_{1}^{\prime}}\left(X^{\prime}\right) \times \cdots \times \operatorname{Sym}^{b_{r}^{\prime}}\left(X^{\prime}\right)\right)
$$

of the fixed point locus $\left(\mathcal{Q}^{\prime}\right)^{T^{\prime}} \subset \mathcal{Q}^{\prime}$ which is isomorphic to $Z$. Now from Proposition 3.1 it follows that

- at least one of the $2 r$ integers $\left\{a_{1}^{\prime}, \cdots, a_{r}^{\prime}, b_{1}^{\prime}, \cdots, b_{r}^{\prime}\right\}$ is one, and

- $X$ is isomorphic to $X^{\prime}$.

This completes the proof. 


\section{ACKNOWLEDGEMENTS}

The first named author thanks Facultad de Matemáticas, PUC Chile, for hospitality. He also acknowledges the support of a J. C. Bose Fellowship. The second named author was partially supported by FONDECYT grant 1150404 during the preparation of this article.

\section{REFERENCES}

[Ba] J.M. Baptista, On the $L^{2}$-metric of vortex moduli spaces, Nucl. Phys. B 844 (2011), 308-333.

[BDW] A. Bertram, G. Daskalopoulos and R. Wentworth, Gromov invariants for holomorphic maps from Riemann surfaces to Grassmannians, Jour. Amer. Math. Soc. 9 (1996) 529-571.

[Bif] E. Bifet, Sur les points fixes schéma $\operatorname{Quot}_{\mathcal{O}_{X} / X, k}$ sous l'action du tore $\mathbf{G}_{m, k}^{r}$, Com. Ren. Math. Acad. Sci. Paris 309 (1989), 609-612.

[BGL] E. Bifet, F. Ghione and M. Letizia, On the Abel-Jacobi map for divisors of higher rank on a curve, Math. Ann. 299 (1994), 641-672.

[BDHW] I. Biswas, A. Dhillon, J. Hurtubise and R. A. Wentworth, A generalized Quot scheme and meromorphic vortices, Adv. Theo. Math. Phys. 19, No. 4 (2015), arXiv:1410.1182 [math.AG].

$[\mathrm{BDH}] \quad$ I. Biswas, A. Dhillon and J. Hurtubise, Automorphisms of the Quot schemes associated to compact Riemann surfaces, Int. Math. Res. Not. (2015), 1445-1460.

[BR] I. Biswas and N. M. Romão, Moduli of vortices and Grassmann manifolds, Comm. Math. Phy. 320 (2013), 1-20.

[Br] S. Bradlow, Vortices in holomorphic line bundles over closed Kähler manifolds, Commun. Math. Phys. 135 (1990), 1-17.

[EINOS] M. Eto, Y. Isozumi, M. Nitta, K. Ohashi and N. Sakai, Solitons in the Higgs phase: the moduli matrix approach, J. Phys. A: Math. Gen. 39 (2006), 315-392.

[Ma] I. G. Macdonald, Symmetric products of an algebraic curve, Topology 1 (1962), 319-343.

School of Mathematics, Tata Institute of Fundamental Research, Homi Bhabha Road, BOMBAY 400005, INDIA

E-mail address: indranil@math.tifr.res.in

Facultad de Matemáticas, PUC Chile, Av. Vicuña Mackenna 4860, Santiago, Chile; Chennai Mathematical Institute, H1, Sipcot it Park, Siruseri, Kelambakkam 603103, INDIA

E-mail address: smehrotra@mat.uc.cl 